\title{
Enhanced Superconductivity in Monolayer
}

\section{$T_{d}-\mathrm{MoTe}_{2}$}

Daniel A. Rhodes, ${ }^{*}, \uparrow, \triangle$ Apoorv Jindal,,$\triangle \triangle$ Noah F. Q. Yuan, ${ }^{\S}$ Younghun Jung, ${ }^{\dagger}$ Abhinandan Antony, ${ }^{\dagger}$ Hua Wang,, Bumho Kim, ${ }^{\dagger}$ Yu-che Chiu, ${ }^{\perp}$ Takashi Taniguchi, \# Kenji Watanabe,\# Katayun Barmak, ${ }^{@}$ Luis Balicas, ${ }^{\perp}$ Cory R. Dean, "Xiaofeng Qian," Liang Fu, ${ }^{\S}$ Abhay N. Pasupathy, ${ }^{*}$, and James Hone,

$\dagger$ Department of Mechanical Engineering, Columbia University, New York, NY 10027 $\ddagger$ Department of Materials Science and Engineering, University of Wisconsin - Madison, Madison, WI 53706

9 Department of Physics, Columbia University, New York, NY, 10027

$\S$ Department of Physics, Massachusetts Institute of Technology, Cambridge, MA 02142 ||Department of Materials Science and Engineering, Texas A\&M University, College Station, Texas 77840

$\perp$ Department of Physics and National High Magnetic Field Laboratory, Florida State University, Tallahassee, FL 32306

\# National Institute for Materials Science, 1-1 Namiki, Tsukuba 305-0044, Japan @ Department of Applied Physics and Applied Mathematics, Columbia University, New York, NY 10027 $\triangle$ These authors contributed equally.

E-mail: darhodes@wisc.edu; apn2108@columbia.edu; jh228@columbia.edu 


\section{Single Crystal Growth}

High quality single crystals were prepared by combining molybdenum powder $(99.999 \%)$ and tellurium lumps $(99.9999+\%)$ in a ratio of 1:20 in a quartz ampoule and subsequently sealing under vacuum $\left(\sim 5 \times 10^{-6}\right.$ Torr $)$. The reagents were then heated to $1100{ }^{\circ} \mathrm{C}$ within $24 \mathrm{~h}$ and held at this temperature for $24 \mathrm{~h}$ before being cooled to $880{ }^{\circ} \mathrm{C}$ over $400 \mathrm{~h}$. At 880 ${ }^{\circ} \mathrm{C}$ the tellurium flux was decanted in a centrifuge and the samples were quenched in air. The obtained $1 \mathrm{~T}^{\prime}-\mathrm{MoTe} \mathrm{T}_{2}$ single crystals were annealed at $425^{\circ} \mathrm{C}$ with a $200{ }^{\circ} \mathrm{C}$ gradient for $48 \mathrm{~h}$ to remove any residual tellurium. As a quality check, batches following this recipe have yielded bulk crystals with a residual resistivity ratio of 600 to 1400 (see Figure S1).

\section{Fabrication}

a. Via Devices: For sample fabrication, between 20 to $30 \mathrm{~nm}$ thick $h$-BN flake is first exfoliated onto $\mathrm{SiO}_{2}$, and holes are etched using a $\mathrm{SF}_{6}$ or $\mathrm{CHF}_{3} / \mathrm{O}_{2}$ plasma mix. A second, larger diameter hole is then opened through a PMMA mask and Pd/Au, (20/50 nm), or Au $(50 \mathrm{~nm})$ is deposited into the holes, forming an embedded contact in the $h$-BN flake that has an overlapping portion, henceforth referred to as a via contact. ${ }^{1}$ This is then picked up by a dry transfer method using polypropylene carbonate (PPC) as the pickup polymer. ${ }^{2}$

Monolayer $T_{d}-\mathrm{MoTe}_{2}\left(1 \mathrm{~L}-\mathrm{MoTe}_{2}\right)$ flakes were exfoliated onto PDMS inside a nitrogenfilled glovebox, identified optically on either PDMS using ImageJ software or by color contrast on $\mathrm{SiO}_{2}$ (see Figure S2). Identified monolayers were then picked up from PDMS or from $\mathrm{SiO}_{2}$ after PDMS transfer using the already picked up via contacts. The $h-\mathrm{BN} / \mathrm{MoTe}_{2}$ stacks were then placed onto a previously Ar- $\mathrm{O}_{2}$ annealed, ${ }^{3}$ between 20 to $30 \mathrm{~nm}$ thick, $h$-BN flake. After encapsulation, the devices were removed from the glovebox and the PPC was subsequently removed by rinsing in chloroform or acetone. Finally, the devices were etched using $\mathrm{O}_{2}-\mathrm{CHF}_{3}$ plasma to isolate monolayers and contacts were fabricated through conventional e-beam lithography (EBL) techniques with either $\mathrm{Ti} / \mathrm{Au}$ or $\mathrm{Cr} / \mathrm{Au}, 5 / 75 \mathrm{~nm}$, 
as the deposited contact to the via contacts. In this case, we deliberately made the overlapping region of the top metal to be larger than the via contact to insure that any gap between the $h$-BN and the via contact caused during the transfer process would be filled and prevent further exposure of the sample to air. Figure S3 depicts the total process flow. Overdoped samples (i.e. increasing $T_{\mathrm{c}}$ with positive $V_{\mathrm{bg}}$ ) were typically produced when only $\mathrm{Si} / \mathrm{SiO}_{2} / h$-BN was used as a backgate, i.e. sample $\mathrm{S} 2$. However, using a local graphite backgate produced the overall highest $T_{\mathrm{c}}$ and shows a pronounced peak in resistivity at low backgate bias, i.e. sample S1. Sample S1 is the sample referred to in the main text.

b. Prepatterned Contact Devices: While prepatterned contacts had less yield over via devices for monolayers, the yield for multilayer devices using prepatterned contact was dramatically higher than that of via contacts. Using $12 \mathrm{~nm} \mathrm{Au}$, or $\mathrm{AuPd}$, prepatterned contacts on $20-30 \mathrm{~nm} h$-BN we were able to achieve nearly $100 \%$ yield of successful devices when proper care was taken into consideration post-stacking. First, metal backgates were patterned on to $285 \mathrm{~nm} \mathrm{SiO}_{2}$ using standard EBL techniques and a bilayer - PMMA 495A4/PMMA 950A2 resist mask. After patterning, $10 \mathrm{~nm}$ of AuPd was deposited via ebeam deposition, followed annealing in vacuum at $300{ }^{\circ} \mathrm{C}$, and a low power $\mathrm{O}_{2}$ plasma to remove any residual polymer on the prepatterned metal backgates. 20-30 nm $h$-BN was then transferred on to the metal backgates using the aforementioned dry stacking method. Subsequently, contacts were patterned on to the $h$-BN using standard EBL and $12 \mathrm{~nm}$ of either Au or AuPd were deposited via e-beam deposition. After liftoff in acetone, contacts were cleaned by atomic force microscopy (AFM) using a Bruker Dimension Icon in contact mode with an Otespa-R3 AFM tip and a deflection voltage ranging from 0.3 to $0.6 \mathrm{~V}$. All subsequent steps were the same as described in section $2 \mathrm{a}$. 


\section{Transport Measurements}

In Fig. S4a. we plot the resistivity versus temperature for samples S1 and S2, where sample $\mathrm{S} 1$ is the sample from the main text. Sample S2 displays a resistivity (multiplied by a factor of 10) of $76 \Omega$. For each sample the mean free path was calculated using either the Drude model: $\sigma=n e^{2} l_{\text {Drude }} / m^{*} v_{\mathrm{F}}$, where $\sigma$ is the conductivity, $n$ is the carrier density, $e$ the charge of an electron, $m^{*}$ the effective mass or through a similar formalism which takes into account the degeneracy of valley and spin: $l_{\operatorname{deg}}=h \sigma /\left(e^{2} \sqrt{g_{s} g_{v} \pi n}\right)$, here $g_{s}=g_{v}=2$ are the spin and valley degeneracy, ${ }^{4}$ respectively. Effective mass was obtained by fitting a first-principles Density functional theory (DFT) calculated band structure along $k_{x}$ and $k_{y}$. The calculated effective mass of the two electron pockets is 0.22 and $0.28 m_{e}$ along $k_{x}$ and $k_{y}$, respectively, while the effective mass of the hole pocket at $\Gamma$ is $0.34 m_{e}$ along $k_{x}$ and $0.64 m_{e}$ along $k_{y}$, indicating the anisotropic nature of $1 \mathrm{~L}-\mathrm{MoTe}_{2}$. In Table $\mathrm{S} 1$, we summarize the values of the mean free path calculated from both models using an average effective mass of $0.37 m_{e}$. However, since 1L-MoTe 2 is semimetallic the exact value of the carrier density used for determining the mean free path is difficult to extract experimentally — showing unrealistically large changes in carrier density as a function of gate induced carrier density (see Figure S5). This is amplified by the difficulty of producing samples with good Hall geometry, as etching deep into the $1 \mathrm{~L}-\mathrm{MoTe}_{2}$ portion of the heterostructure causes rapid sample degradation.

Given these complications, we evaluated the mean free path assuming a carrier density, $n_{\text {DFT }}$, extracted from the DFT calculated Fermi surfaces (FS), which totals to $\sim \times 10^{13} / \mathrm{cm}^{2}$, where a single hole pocket has a higher carrier concentration of $5.45 \times 10^{12} \mathrm{~cm}^{-2}$ as compared to that of the electrons $\left(2.25 \times 10^{12} \mathrm{~cm}^{-2}\right.$ each $)$. While we have not verified directly the carrier density of the monolayer directly, the validity of this assumption can be verified through transport measurements of bilayer, and trilayer samples where Shubnikov-de Haas (SdH) oscillations are visible in the magnetoresistance (see Figure S6). Shown in Figure S6b, both the bilayer and trilayer samples exhibit superlinear behavior for the normal state resistance as a function of the applied magnetic field, suggested a nearly charged compensated density 
of electron and holes. Dashed lines are fits to the function: $R_{\mathrm{xx}}=R_{0}+\gamma B^{\alpha}$, where $\gamma$ represents a combination of sample geometry, mobility, and carrier density and $R_{0}$ is the typical $(l / w)\left[n e\left(\mu_{\mathrm{e}}+\mu_{\mathrm{h}}\right)\right]^{-1}$. For perfect compensation, $\alpha$ is expected to be 2 , whereas for single dominant carrier $\alpha=0$. Shown in Figure S6b, fitting to the trilayer sample yields a value of 1.71, and to the bilayer 1.56. Deviations from a value of 2 can either indicate a slightly larger carrier population for either holes (as suggested by DFT) or electrons, an anisotropic FS, nontrivial FS topology, or disorder. ${ }^{5}$ In this case, it's likely a combination of disorder and slight offset from perfect compensation given the trilayer has a value closer to 2 than that of the bilayer and concomitantly has a higher mobility, which is estimated from the onset of the $\mathrm{SdH}$ oscillations $\left(950 \mathrm{~cm}^{2} / \mathrm{Vs}\right.$ for the trilayer, $850 \mathrm{~cm}^{2} / \mathrm{Vs}$ for the bilayer). Background subtraction, using a third degree polynomial, of the magnetoresistance yields an SdH signal in both the bilayer and trilayer with a frequency of 412-449 $\mathrm{T}$. Using the frequency of the oscillations with respect to the inverse of the magnetic field $\left(B_{\mathrm{F}}\right)$ in combination with the Onsager relation for magnetic field, $B_{\mathrm{F}}=\phi_{0} \mathrm{~A} / 2 \pi^{2}$, where $\phi_{0}$ is the magnetic flux quantum, and assuming a 2D, circular Fermi surface, $\mathrm{A}=\pi k_{\mathrm{F}}^{2}, k_{\mathrm{F}}=\sqrt{2 \pi n}$ yields a carrier density of $\sim 2 \times 10^{13} \mathrm{~cm}^{-2}$ - in reasonably good agreement with the DFT calculated carrier density. Given that only one frequency is able to be resolved and the effective mass of the electrons is nearly half that of holes, and thus twice as mobile, this peak is likely to be the electron pockets.

Using the aforementioned carrier density, the mean free paths are calculated for samples S1 and S2 and summarized in Table S1. For sample S2, the clean limit is easily achieved as the mean free path $(307 \mathrm{~nm})$ is twenty times larger than that of the coherence length (14-16 nm). S1, on the other hand, has a much smaller mean free path on the order of the coherence length at zero gate voltage. However, as indicated by the unrealistic spin-orbit scattering times (see Table S2), we are still able to fit our spin-orbit coupling model for $H_{\mathrm{c} 2}^{\|}$ vs $T$ (see below) and extract values for the spin-orbit coupling strengths in- and out-of-plane in general agreement with sample S2. 


\section{Fitting of experimental upper critical field on 1L-}

\section{$\mathrm{MoTe}_{2}$}

Klemm-Luther-Beasley: The KLB model is defined by a fit to $\ln \left(T / T_{c}\right)+\Psi\left(\frac{1}{2}+\frac{3 \tau_{\mathrm{SO}} \mu_{B}^{2} H_{\mathrm{c} 2}^{2}}{4 \pi \hbar k_{B} T}\right)-$ $\Psi\left(\frac{1}{2}\right)=0$, where $\Psi$ is the digamma function, $\tau_{S O}$ the spin-orbit scattering time, and $\mu_{B}$ is the Bohr magneton. With in- and out-of-plane SOC: For a paramagnetic limiting field at finite temperature, $T$, we consider the linearized gap equation:

$\log \left(T / T_{\mathrm{c}}\right)+\left\langle w_{\boldsymbol{k},+} \Phi\left(\rho_{\boldsymbol{k},+}\right)+w_{\boldsymbol{k},-} \Phi\left(\rho_{\boldsymbol{k},-}\right)=0\right\rangle_{\mathrm{FS}}$, with

$w_{ \pm}=\frac{1}{2}\left[1 \pm \frac{|\boldsymbol{g}|^{2}-|\mu \boldsymbol{B}|^{2}}{|\boldsymbol{g}+\mu \boldsymbol{B}| \cdot|\boldsymbol{g}-\mu \boldsymbol{B}|}\right], \rho_{ \pm}=\frac{|\boldsymbol{g}+\mu \boldsymbol{B}| \mp|\boldsymbol{g}-\mu \boldsymbol{B}|}{2 \pi T}, \Phi(x)=\operatorname{Re}\left\{\psi\left(\frac{1+i x}{2}\right)-\psi\left(\frac{1}{2}\right)\right\}$.

Where $\boldsymbol{g}$ is the spin-orbit field, $\boldsymbol{B}$ is the magnetic field, $\mu$ is the electron magnetic moment, and $\langle\ldots\rangle_{\mathrm{FS}}$ is the Fermi surface average: $\left\langle f_{\boldsymbol{k}}\right\rangle_{\mathrm{FS}} \equiv \frac{1}{N_{0}} \int_{\xi_{\boldsymbol{k}}=0} \frac{d^{2} \boldsymbol{k}}{(2 \pi)^{2}} f_{\boldsymbol{k}}$, where $\xi_{\boldsymbol{k}}$ is the kinetic energy and $\xi_{\boldsymbol{k}}=0$ is the Fermi surface. We can then fit the upper critical field data using $\boldsymbol{g}_{\boldsymbol{k}}=\left(\alpha_{y} k_{y}, \alpha_{x} k_{x}, \alpha_{I} k_{y}\right)$. For an in-plane field $\boldsymbol{B}=B(\cos \phi \hat{\boldsymbol{x}}+\sin \phi \hat{\boldsymbol{y}})$ we have:

$\left\langle\left|\boldsymbol{g}_{\boldsymbol{k}} \times \hat{\boldsymbol{B}}\right|^{2}\right\rangle_{\mathrm{FS}}^{1 / 2}=\sqrt{\left(\alpha_{y}^{2}+\alpha_{I}^{2} \sin ^{2} \phi\right) K_{y}^{2}+\alpha_{x}^{2} K_{x}^{2} \cos ^{2} \phi}$

and $\left\langle\left|\boldsymbol{g}_{\boldsymbol{k}} \cdot \hat{\boldsymbol{B}}\right|^{2}\right\rangle_{\mathrm{FS}}^{1 / 2}=\sqrt{\alpha_{y}^{2} K_{y}^{2} \cos ^{2} \phi+\alpha_{x}^{2} K_{x}^{2} \sin ^{2} \phi}, K_{x, y} \equiv\left\langle k_{x, y}^{2}\right\rangle_{\mathrm{FS}}^{1 / 2}$. In the main text, we have defined $\Delta_{\mathrm{SO}}^{\|} / \Delta_{\mathrm{so}}^{\perp}=\frac{\left\langle\left|g_{k} \cdot \hat{\boldsymbol{B}}\right|^{2}\right\rangle_{\mathrm{FS}}^{1 / 2}}{\left\langle\left|\boldsymbol{g}_{k} \times \hat{\boldsymbol{B}}\right|^{2}\right\rangle_{\mathrm{FS}}^{1 / 2}}$

Using this formalism, we fit samples one and two from the main text to reconfirm the differing spin-orbit coupling, shown in Figure S8. From Figure S8a., S1 shows a considerable in plane component of spin-orbit coupling (SOC). In order to evaluate the accuracy of these fits, we attempt to fit several other values for the spin-orbit coupling strengths in- and out-ofplane. As shown, small deviations in the overall in-plane spin-orbit coupling strength show considerable differences, from overestimation at low in-plane values, to an uncharacteristic rollover and underestimation at substantially higher in-plane SOC. As such, our best fit gives a relative coupling strength of in-plane SOC as compared to out-of-plane SOC, $\Delta_{\text {SOC }}^{\|} / \Delta_{\text {SOC }}^{\perp}$, 
of $15.3 \%$. However, in the case of samples where $H_{\mathrm{c} 2}^{\|}$is only measured down to values of $T / T_{\mathrm{c}} \sim .5$ with larger out-of-plane SOC the uncertainty in the fitting becomes substantially larger. In sample S2 (see Figure S8b.), where this is indeed the case, we find a $\Delta_{\text {SOC }}^{\|} / \Delta_{\text {SOC }}^{\perp}$ with values between 0 to $7.5 \%$ fits reasonably well, but values larger than $7.5 \%$ repeatedly underestimate the experimental values between $T=2.0 \mathrm{~K}$ and $T=3.5 \mathrm{~K}$. Overall, the two samples are in qualitative agreement with the expectation of an anisotropic in-plane magnetic field dependence as described in the main text.

\section{First-principles electronic structure calculations}

The ground-state crystal structure of monolayer $\mathrm{MoTe}_{2}$ was obtained from first-principles density functional theory (DFT) calculations as implemented in the Vienna Ab initio Simulation Package (VASP) using the plane wave basis ${ }^{6}$ with an energy cutoff of $400 \mathrm{eV}$ and the projector-augmented wave method. ${ }^{7}$ We adopted the Perdew-Burke-Ernzerhof's form of exchange-correlation functional within the generalized-gradient approximation and the Monkhorst-Pack k-point sampling of $8 \times 16 \times 1$ for the integration over the first Brillouin zone as well as a vacuum slab of $16 \AA$ along the $\mathrm{z}$ direction to reduce image interaction under the periodic boundary condition in the calculation. The monolayer crystal structure was fully relaxed with a maximum residual force of less than $0.01 \mathrm{eV} / \AA$. To investigate the detailed electronic structure, we performed DFT calculations with a hybrid HSE06 exchange-correlation functional $^{8}$ and transformed the DFT Kohn-Sham eigenstates into a set of highly localized quasiatomic orbitals and corresponding first-principles tight-binding Hamiltonian. ${ }^{9,10}$ The Hamiltonian was further symmetrized using WannierTools. ${ }^{11}$ Using the effective Hamiltonian we computed the corresponding fine electronic band structure and spin texture in the 2-D Brillouin zone. An electric field of $0.01 \mathrm{~V} / \AA$ was applied along the $\mathrm{z}$ direction which breaks the $\mathrm{C}_{2}$ two-fold rotation symmetry, thus breaking the inversion symmetry and leading to the spin splitting which qualitatively matches the experimental data. 

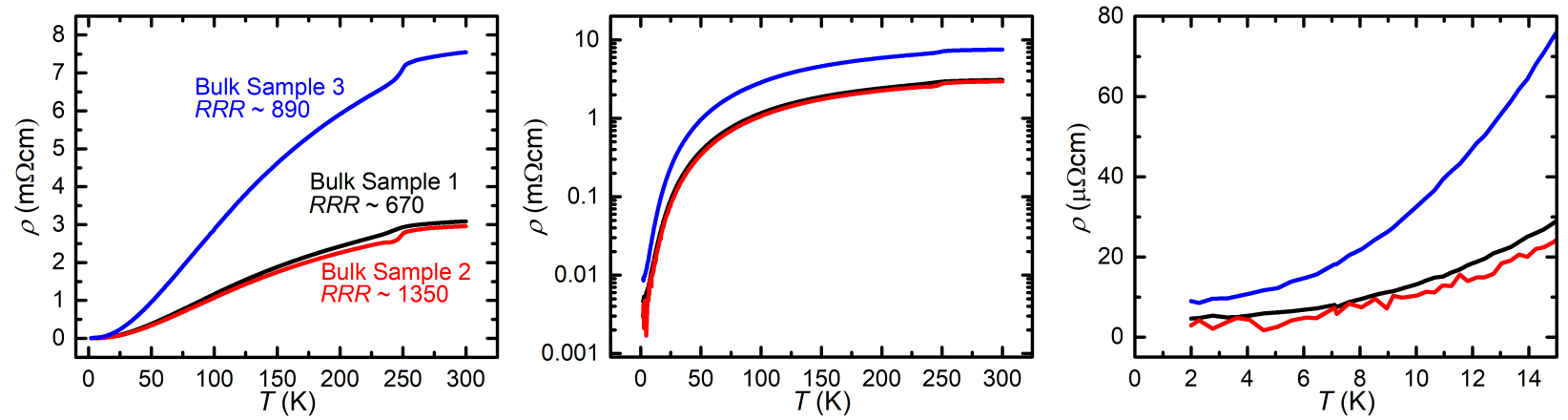

Figure S1 | Bulk Crystal Quality. a-b, Temperature dependence of the resistance for three randomly chosen crystals from three different batches all grown the same as the crystals use for exfoliation of the devices in the main text, b is in log scale. c, Zoom-in of $\mathbf{a}$ to highlight there is no superconducting transition down to $2 \mathrm{~K}$. 

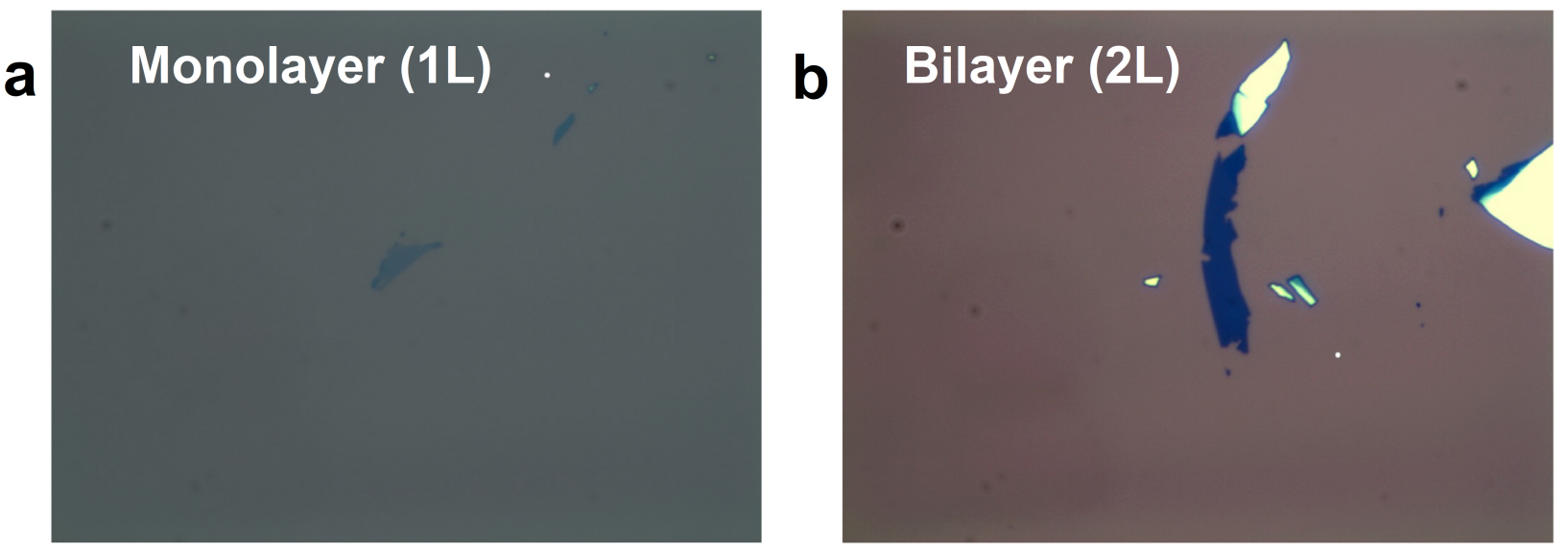

C Trilayer (3L)
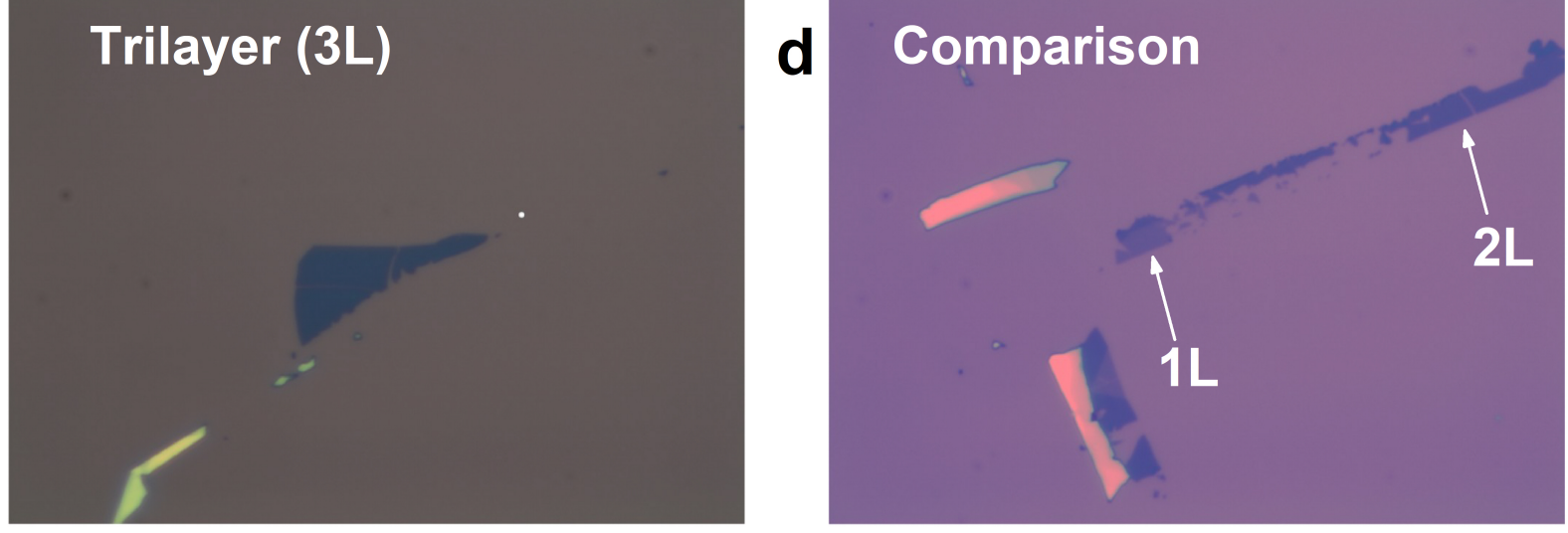

Figure S2 | Optical contrast. a-d, Optical images of few-layer $T_{d}-\mathrm{MoTe}_{2}$. All images are approximately $120 \times 120 \mu \mathrm{m}^{2}$ in area. 

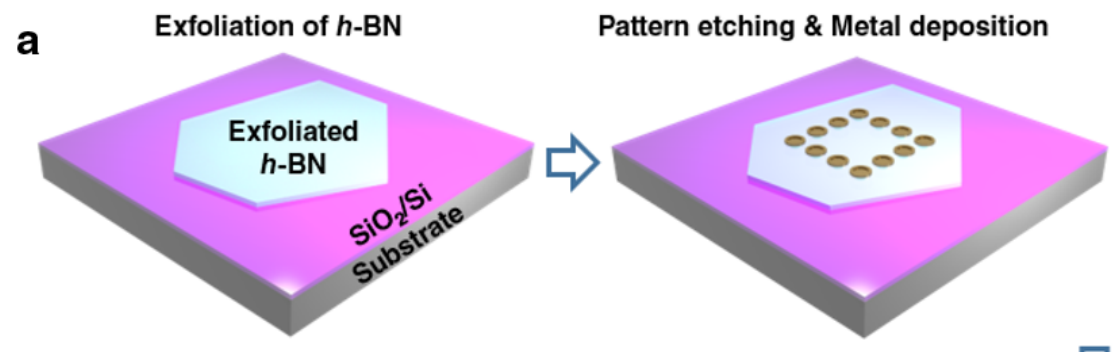

Transfer the stack on the bottom $h$-BN
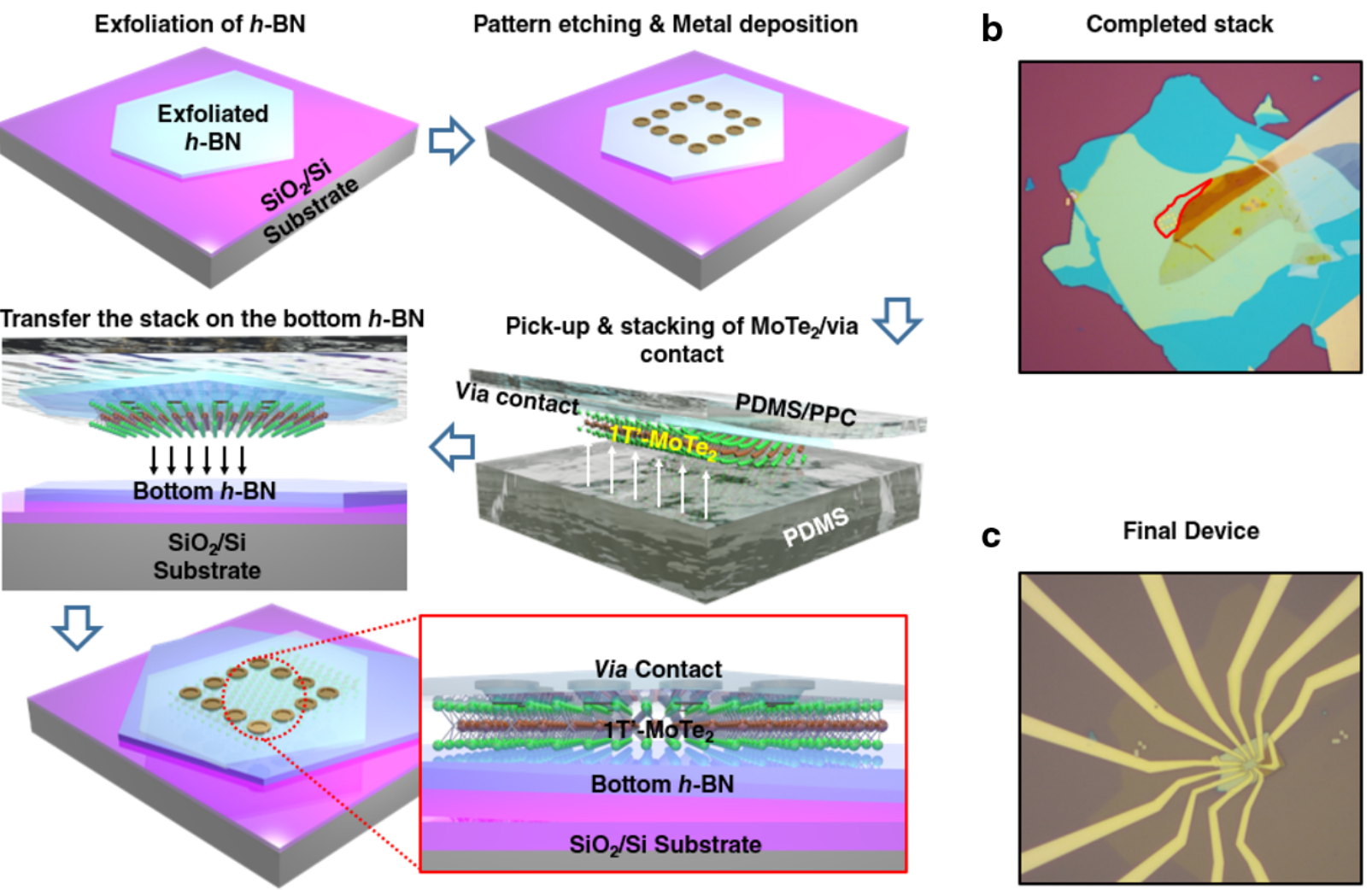

Figuer S3 | Device Fabrication. a, Depiction of the initial via fabrication and stacking sequence. b, Optical image of a completed stack, monolayer portion is outlined in red. c, Optical image of a completed device. 

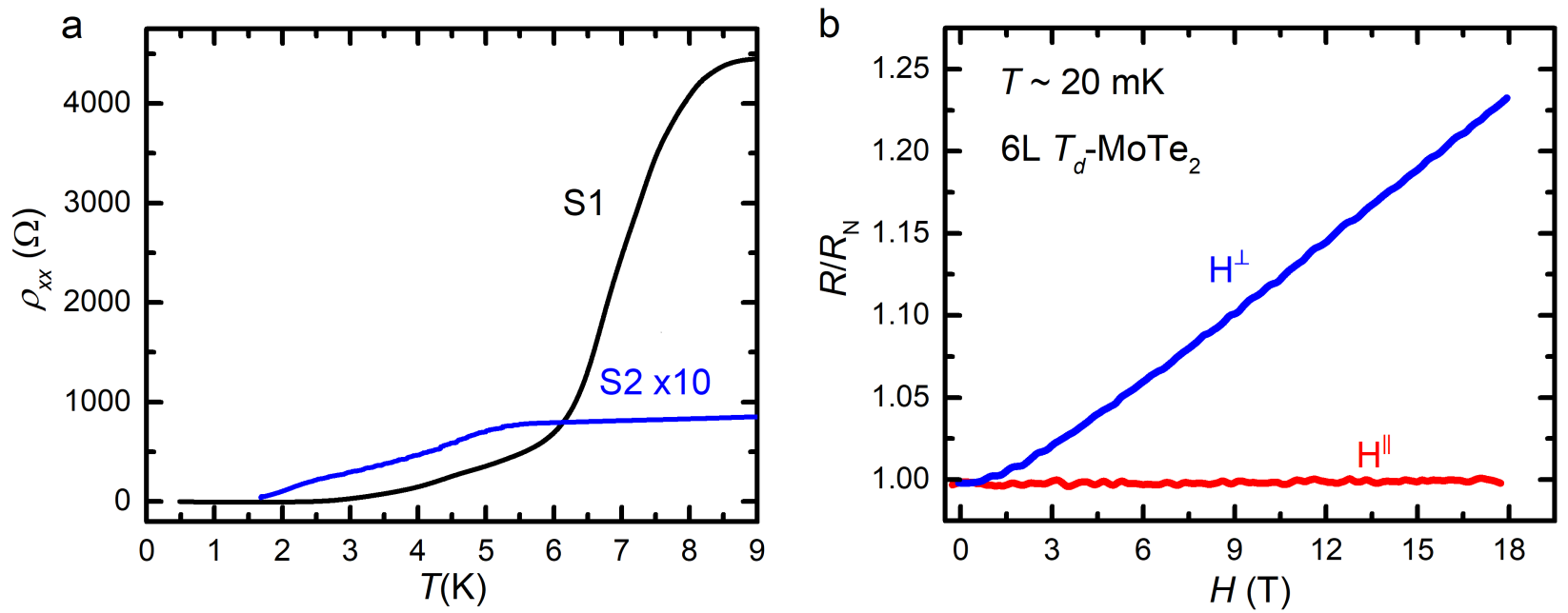

Figure S4 | Temperature dependence of normalized resistivity for various thicknesses of $T_{d}-\mathrm{MoTe}_{2}$. a, Low temperature $T$-dependence of two monolayer samples, S1 and $\mathrm{S} 2$, both showing enhanced $T_{\mathrm{c}}$. S1 is a graphite backgated sample, whereas $\mathrm{S} 2$ utilizes a silicon backgate. b, Parallel and perpendicular magnetic field dependence for a 6-layer sample of $T_{d}-\mathrm{MoTe}_{2}$ at $T=20 \mathrm{mK}$. 

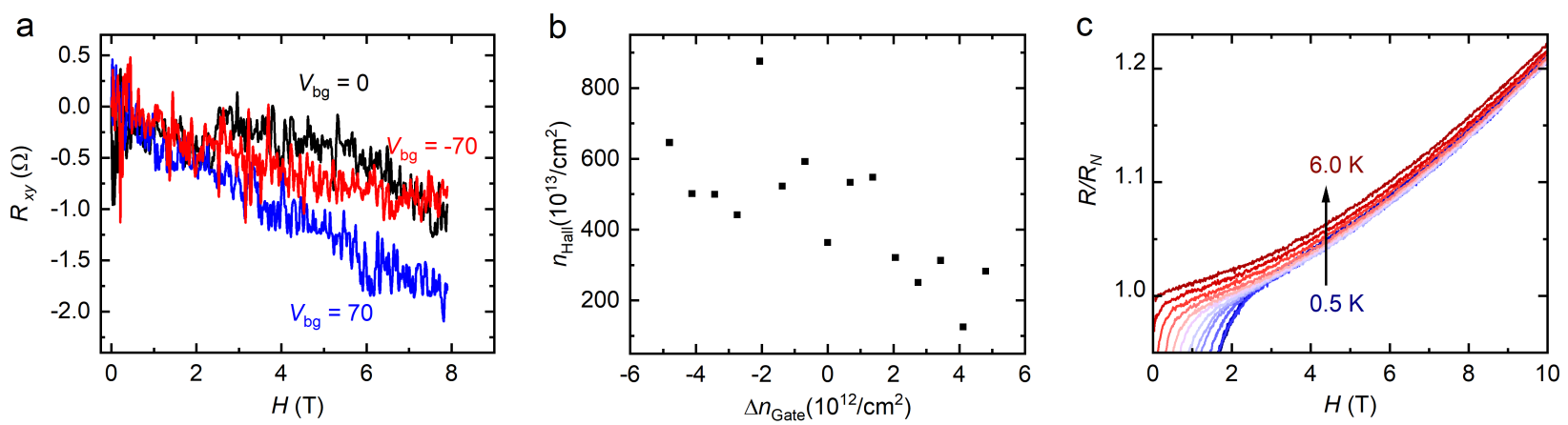

Figure S5 | Semimetallic behavior. a, Anti-symmetrized Hall signal for a monolayer sample of $\mathrm{MoTe}_{2} \mathbf{b}$, Experimental single carrier density, $n_{\text {Hall }}$, vs. gate induced carrier density, $\Delta n_{\text {Gate }}$. c, Normalized Magnetoresistance as a function of field above $H_{\mathrm{c} 2}^{\perp}$ for sample $\mathrm{S} 2$ and for $V_{\mathrm{bg}}=0 \mathrm{~V}$. Notice the nonlinear behavior. 

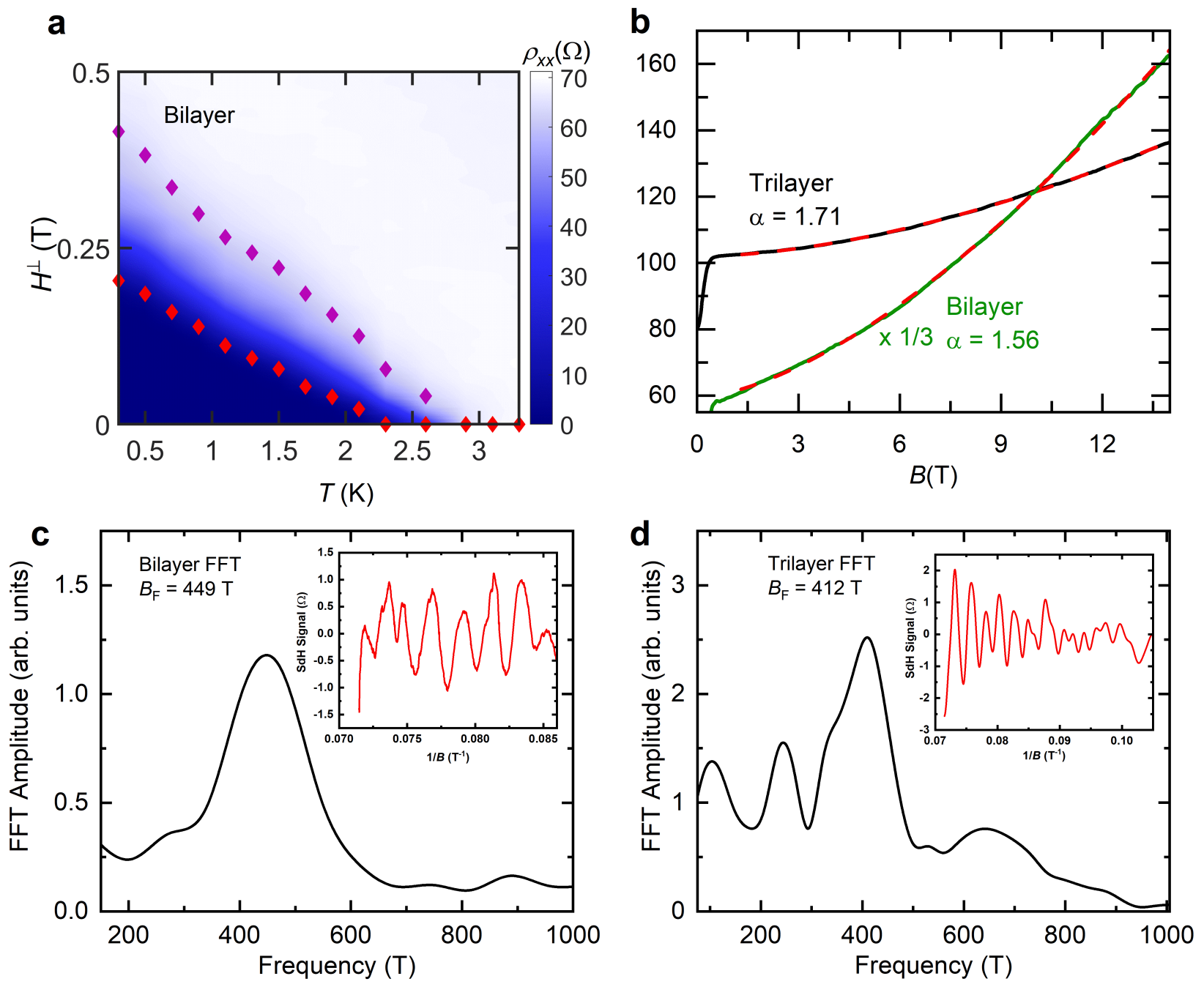

Figure S6 | Bilayer and Trilayer Behavior. a, Perpendicular magnetic field vs temperature phase diagram for a bilayer sample with $T_{\mathrm{c}}=2.5 \mathrm{~K}$, where a coherence length of $\sim 30 \mathrm{~nm}$ can be extracted. $\mathbf{b}, R_{\mathrm{xx}}$ as a function of the magnetic field in the perpendicular direction, dashed lines are fits the polynomial function described in the text. $\mathbf{c}, \mathbf{d}$ The fast Fourier transform of the $\mathrm{SdH}$ signal as extracted from (b) for the bilayer and trilayer sample, respectively. Inset c,d: the $\mathrm{SdH}$ signals for both the bilayer and trilayer samples, respectively 


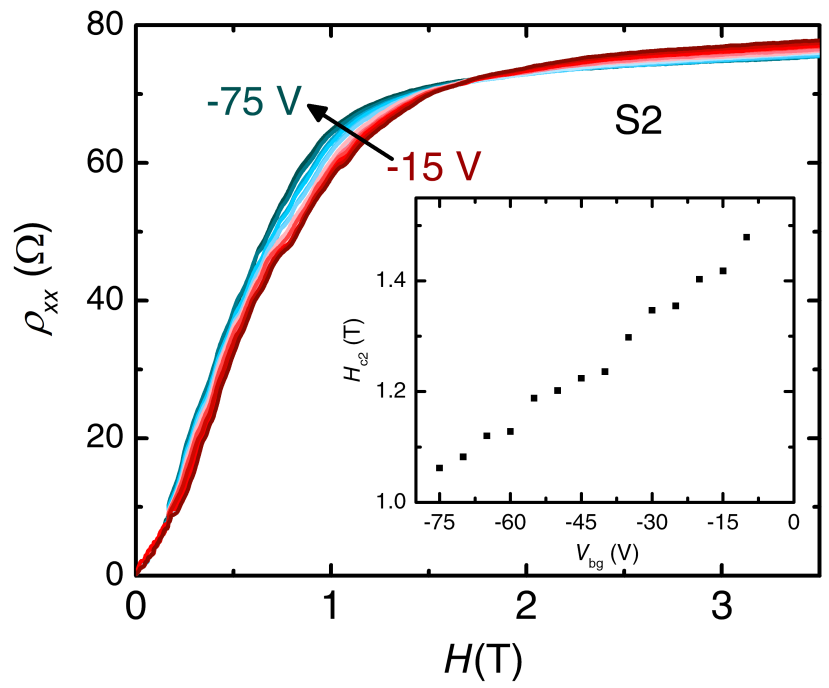

Figure S7 $\mid \Delta n_{\text {Gate }}$. Resistivity vs field and for several values of gate voltage applied to sample $\mathrm{S} 2$ at $T=250 \mathrm{mK}$. Notice that the sheet resistance in the normal state decreases monotonically as a function of increasing negative gate voltages. Inset: $H_{\mathrm{c} 2}^{\perp}$ monotonically increasing as a function of the backgate voltage. 

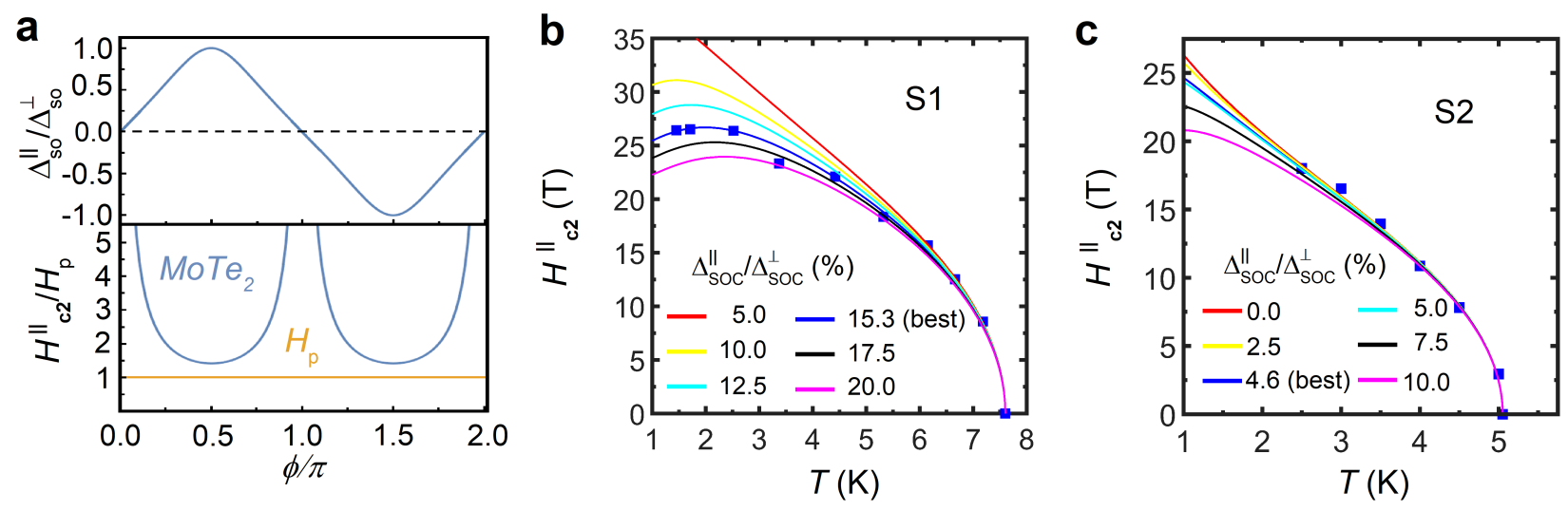

Figure S8 | Spin-orbit coupling fittings. a-b, Measured $H_{\mathrm{c} 2}^{\|}$for fields parallel to the $a b$-plane for Samples 1, and 2 with fits to several other relative spin-orbit coupling strengths for reference. Blue squares are experimental data, solid lines are theoretical fits using the formalism described in the text. 


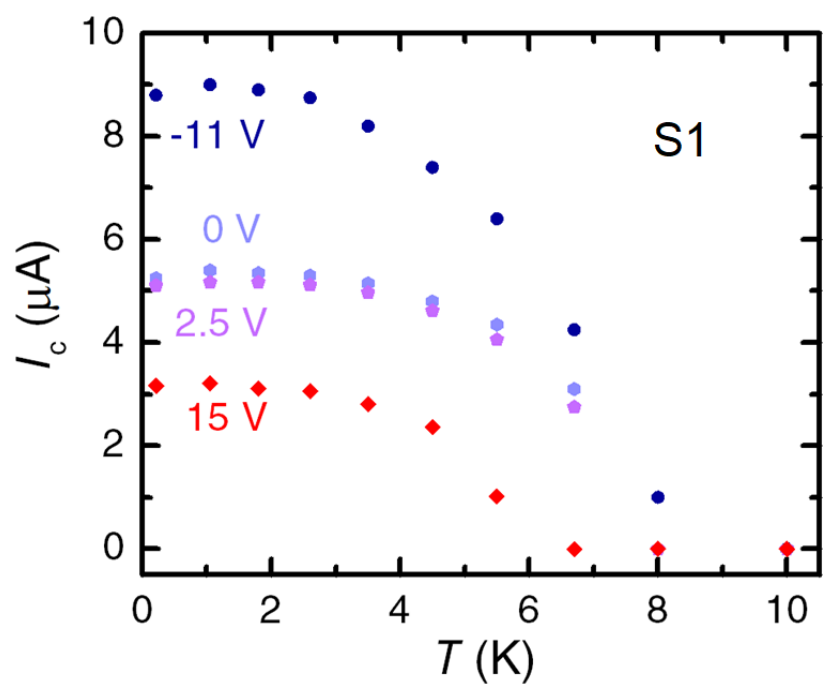

Figure S9 $\mid$ Critical current. Critical current, $I_{\mathrm{c}}$ as a function of temperature and varying gate voltage for sample S1 described in the main text. As in the case of the critical temperature, $I_{\mathrm{c}}$ increases monotonically as the gate voltage is decreases. 
Table 1. Summary of the mean free paths for each sample. The mean free paths are either derived through the Drude model for the value of $n$ given by the SdH oscillations $\left(n \sim 2 \times 10^{13} / \mathrm{cm}^{2}\right)$ or by the model which takes into account valley and spin degeneracy given in the text.

\begin{tabular}{|l||l|l|l|l|l|}
\hline & $\begin{array}{l}T_{\mathrm{c}} \\
(\mathrm{K})\end{array}$ & $\xi_{0}(n m)$ & $\begin{array}{l}\rho_{x x} \\
(\Omega)\end{array}$ & $\begin{array}{l}l_{\text {Drude }} \\
(\mathrm{nm})\end{array}$ & $l_{\operatorname{deg}}(\mathrm{nm})$ \\
\hline S1 & 7.6 & 7.5 & 3560 & 6.73 & 4.76 \\
\hline S2 & 5 & 13.9 & 78 & 307 & 217 \\
\hline
\end{tabular}


Table 2. Summary of scattering rates. The scattering rates are either derived through the Drude model for the calculated value of $v_{\mathrm{F}}$ and $n$ from the SdH oscillations $(n \sim 2 \times$ $\left.10^{13} / \mathrm{cm}^{2}\right)$ and deriving $v_{\mathrm{F}}$ assuming a 2-D circular Fermi surface, i.e. $v_{\mathrm{F}}=\hbar \sqrt{2 \pi n} / \mathrm{m}^{*}$ and using the values of the mean free path outlined in Table $\mathrm{S} 1$ for $\tau_{\text {deg }}$. The values for the spin-orbit coupling are taken from the best fits outlined in Fig. S5.

\begin{tabular}{|l||l|l|l|l|l|}
\hline & $\begin{array}{l}\tau_{\text {Drude }} \\
(\mathrm{fs})\end{array}$ & $\tau_{\text {deg }}(\mathrm{fs})$ & $\tau_{\text {SOS }}(\mathrm{fs})$ & $\begin{array}{l}\Delta^{\|} \\
(\mathrm{meV})\end{array}$ & $\begin{array}{l}\Delta^{\perp} \\
(\mathrm{meV})\end{array}$ \\
\hline S1 & 54 & 10 & $88-101$ & 0.358 & 2.34 \\
\hline S2 & 2463 & 1742 & $89-150$ & 0.057 & 1.51 \\
\hline
\end{tabular}




\section{References}

\section{References}

(1) Telford, E. J.; Benyamini, A.; Rhodes, D.; Wang, D.; Jung, Y.; Zangiabadi, A.; Watanabe, K.; Taniguchi, T.; Jia, S.; Barmak, K.; Pasupathy, A. N.; Dean, C. R.; Hone, J. Via Method for Lithography Free Contact and Preservation of 2D Materials. Nano Letters 2018, 18, 1416-1420.

(2) Wang, L.; Meric, I.; Huang, P. Y.; Gao, Q.; Gao, Y.; Tran, H.; Taniguchi, T.; Watanabe, K.; Campos, L. M.; Muller, D. A.; Guo, J.; Kim, P.; Hone, J.; Shepard, K. L.; Dean, C. R. One-dimensional electrical contact to a two-dimensional material. Science (New York, N.Y.) 2013, 342, 614-617.

(3) Garcia, A. G. F.; Neumann, M.; Amet, F.; Williams, J. R.; Watanabe, K.; Taniguchi, T.; Goldhaber-Gordon, D. Effective Cleaning of Hexagonal Boron Nitride for Graphene Devices. Nano Letters 2012, 12, 4449-4454.

(4) Fei, Z.; Palomaki, T.; Wu, S.; Zhao, W.; Cai, X.; Sun, B.; Nguyen, P.; Finney, J.; Xu, X.; Cobden, D. H. Edge conduction in monolayer $\mathrm{WTe}_{2}$. Nature Physics 2017, 13, $677-682$.

(5) Mitra, S.; Kang, J. G. S.; Shin, J.; Ng, J. Q.; Sunku, S. S.; Kong, T.; Canfield, P. C.; Shastry, B. S.; Sengupta, P.; Panagopoulos, C. Quadratic to linear magnetoresistance tuning in TmB 4. Physical Review B 2019, 99, 045119.

(6) Kresse, G.; Furthmüller, J. Efficient iterative schemes for ab initio total-energy calculations using a plane-wave basis set. Phys. Rev. B 1996, 54, 11169-11186.

(7) Blöchl, P. E. Projector augmented-wave method. Phys. Rev. B 1994, 50, 17953-17979. 
(8) Heyd, J.; Scuseria, G. E.; Ernzerhof, M. Hybrid functionals based on a screened Coulomb potential. J. Chem. Phys. 2003, 118, 8207-8215.

(9) Qian, X.; Li, J.; Qi, L.; Wang, C.-Z.; Chan, T.-L.; Yao, Y.-X.; Ho, K.-M.; Yip, S. Quasiatomic orbitals for ab initio tight-binding analysis. Phys. Rev. B 2008, 78, 245112.

(10) Mostofi, A. A.; Yates, J. R.; Pizzi, G.; Lee, Y.-S.; Souza, I.; Vanderbilt, D.; Marzari, N. An updated version of wannier90: A tool for obtaining maximally-localised Wannier functions. Computer Physics Communications 2014, 185, 2309-2310.

(11) Wu, Q.; Zhang, S.; Song, H.-F.; Troyer, M.; Soluyanov, A. A. WannierTools: An open-source software package for novel topological materials. Computer Physics Communications 2018, 224, 405-416. 